\title{
Genome sequences published outside of Standards in Genomic Sciences, October - November 2011
}

\author{
Oranmiyan W. Nelson ${ }^{1}$ and George M. Garrity ${ }^{1}$ \\ ${ }^{1}$ Editorial Office, Standards in Genomic Sciences and Department of Microbiology, \\ Michigan State University, East Lansing, MI, USA
}

The purpose of this table is to provide the community with a citable record of publications of ongoing genome sequencing projects that have led to a publication in the scientific literature. While our goal is to make the list complete, there is no guarantee that we may have omitted one or more publications appearing in this time frame. Readers and authors who wish to have publications added to subsequent versions of this list are invited to provide the bibliographic data for such references to the SIGS editorial office.

\section{Phylum Crenarchaeota}

Thermoproteus tenax, strain Kra1, DSM 2078 ${ }^{\mathrm{T}}$ sequence accession FN869859 [1]

Phylum Euryarchaeota

Haloarcula hispanica CGMCC 1.2049, sequence accession CP002921 (chromosome I), CP002922 (chromosome II), and CP002923 (plasmid pHH400) [2]

Methanococcus maripaludis, strain X1 (unculturable) sequence accession CP002913 [3]

\section{Phylum Proteobacteria}

Acinetobacter baumannii strain 1656-2, sequence accession CP001921 [4]

Arcobacter butzleri strain ED-1, sequence accession AP012047, AP012048, and AP012049 [5]

Brucella suis strain 1330, sequence accession CP002997 and CP002998 [6]

Campylobacter fetus subsp. venerealis NCTC 10354, sequence accession AFGH01000000 [7]

“Chromobacterium sp.” strain C-61, sequence accession CAEE01000001 to

CAEE01001118 [8]

Cronobacter sakazakii strain E899, sequence accession AFM000000000 [9]

“Desulfovibrio sp.” strain A2, sequence accession AGFG01000000 [10]

“Erythrobacter sp.” strain NAP1, sequence accession NZ_AAMW00000000 [11]

Escherichia coli strain XH140A, sequence accession AFVX01000000 [12]

Escherichia coli strain XH001, sequence accession AFYG01000000 [13]

Haemophilus haemolyticus strain M19107, sequence accession AFQN00000000 [14]

Haemophilus haemolyticus strain M19501, sequence accession AFQ000000000 [14]

Haemophilus haemolyticus strain M21127, sequence accession AFQP00000000 [14] 
Haemophilus haemolyticus strain M21621, sequence accession AFQQ00000000 [14] Haemophilus haemolyticus strain M21639, sequence accession AFQR00000000 [14] Idiomarina sp." strain A28L, sequence accession AFP001000001 to AFP001000028 [15]

Ketogulonicigenium vulgare" strain WSH-001, sequence accession CP002018 (chromosome), CP002019 (plasmid pKVU_100), and CP002020 (plasmid pKVU_200) [16]

Methylobacter tundripaludum strain SV96, sequence accession AEGW00000000 [17]

Pseudogulbenkiania sp." strain NH8B, sequence accession AP012224 [18]

Pseudomonas aeruginosa NCGM1179, sequence accession DF126593 through

DF126613 [19]

Pseudomonas putida strain B001, sequence accession CAED01000001 to CAEE01000262 [20]

Pseudomonas putida strain B6-2, sequence accession AGCS01000000 [21]

Pseudomonas stutzeri CGMCC 1.1803, sequence accession CP002881 [22]

Ralstonia solanacearum phylotype IB, strain Y45, sequence accession AFWL01000000 [23]

Rheinheimera sp." strain A13L, sequence accession AFHI01000001 through AFHI01000072 [24]

Sphingobium yanoikuyae strain XLDN2-5, sequence accession AFXE01000000 [25]

Vibrio cholerae strain Amazonia, sequence accession AFSV01000000 [26]

\section{Phylum Firmicutes}

Bacillus coagulans strain XZL4, sequence accession AFWM01000000 [27]

Bacillus megaterium strain WSH-002, sequence accession CP003017 (chromosome), plasmids CP003018 (plasmid pBME_100), CP003019 (plasmid pBME_200), and CP003020 (plasmid pBME_300) [28]

Bacillus pumilus strain S-1, sequence accession AGBY00000000 [29]

“Desulfosporosinus sp." strain OT, sequence accession AGAF01000000 [30]

Lentibacillus jeotgali strain Grbi, sequence accession AGAV01000000 [31]

Leuconostoc carnosum KCTC 3525, sequence accession BACM01000000 [32]

Listeria ivanovii subsp. ivanovii strain PAM 55, sequence accession FR687253 [33]

Paenibacillus riograndensis strain SBR5, sequence accession AGBD01000000 [34]

Sporolactobacillus inulinus strain CASD, sequence accession AFVQ00000000 [35]

Streptococcus pseudopneumoniae strain IS7493, sequence accession CP002925 and CP002926 [36]

Streptococcus salivarius strain 57.I, sequence accession CP002888 and CP002889 [37] Streptococcus salivarius strain M18, sequence accession AGBV01000000 [38] 
Streptococcus suis SS12, sequence accession CP002640 [39]

Streptococcus suis D9, sequence accession CP002641 [39]

Streptococcus suis D12, sequence accession CP002644 [39]

Streptococcus suis ST1, sequence accession CP002651 [39]

Weissella thailandensis strain fsh4-2, sequence accession HE575133 through

HE575182 [40]

\section{Phylum Tenericutes}

Mycoplasma anatis strain 1340, sequence accession AFVJ00000000 [41]

Mycoplasma capricolum subsp. capripneumoniae strain M1601, sequence accession AENG01000000 [42]

Mycoplasma putrefaciens Type strain KS1, sequence accession CP003021 [43]

Corynebacterium pseudotuberculosis strain PAT10, sequence accession CP002924 [44]

\section{Phylum Actinobacteria}

Bifidobacterium animalis subsp. lactis strain BLC1, sequence accession CP003039 [45]

Bifidobacterium breve strain DPC 6330, sequence accession AFXX01000000 [46]

Brachybacterium squillarum strain M-6-3, sequence accession AGBX01000000 [47]

“Citricoccus sp.” strain CH26A, sequence accession AFXQ01000000 [48]

Corynebacterium glutamicum strain S9114, sequence accession AFYA01000000 [49]

Dietzia alimentaria strain 72, sequence accession AGFF01000000 [50]

Mycobacterium colombiense CECT 3035, sequence accession AFVW00000000 [51]

Mycobacterium tuberculosis NCGM2209, sequence accession DF126614 and

DF126615 [52]

Rhodococcus erythropolis strain XP, sequence accession AGCF01000000 [53]

Serinicoccus profundi MCCC 1A05965T, sequence accession AFYF00000000 [54]

\section{Phylum Spirochaetes}

Leptospira interrogans, sequence accession CP001221 (CI), CP001222 (CII) [55]

\section{Phylum Bacteroidetes}

Bacteroides faecis Type strain MAJ27T', sequence accession AGDG01000000 [56]

Bizionia argentinensis, Type strain JUB59T sequence accession AFXZ01000000 [57]

Flavobacterium branchiophilum strain FL-15, sequence accession FQ859183 [58]

“Flavobacteriaceae" strain S85, sequence accession AFPK00000000 [59]

\section{Phylum Thermotogae}

“Thermotoga sp.” strain RQ2, sequence accession CP000969 [60] 


\section{Non-Bacterial genomes}

Aspergillus kawachii IF0 4308, sequence accession DF126447 through DF126592,

BACL01000001 through BACL01001641, AP012272 [61]

Cajanus cajan pigeonpea, sequence accession PRJNA72815 [62]

Coxsackievirus A22, sequence accession JN542510 [63]

Gordonia phage GRU1, sequence accession JF923797 [64]

Gordonia phage GTE5, sequence accession JF923796 [64]

Heterocephalus glaber naked mole rat, sequence accession AFSB00000000, AFSB01000000 [65]

Human Adenovirus Prototype 17, sequence accession HQ910407 [66]

Macaca mulatta lasiota rhesus macaque, sequence accession AEHL00000000 [67]

Macaca mulatta mulatta rhesus macaque, sequence accession AEHK00000000 [67]

Porcine epidemic diarrhea virus, sequence accession JN547228 [68]

\section{References}

1. Siebers B, Zaparty M, Raddatz G, Tjaden B, Albers SV, Bell SD, Blombach F, Kletzin A, Kyrpides N, Lanz C, et al. The Complete Genome Sequence of Thermoproteus tenax: A Physiologically Versatile Member of the Crenarchaeota. PLOS ONE 2011; 6:e24222. PubMed doi:10.1371/journal.pone.0024222

2. Liu H, Wu Z, Li M, Zhang F, Zheng H, Han J, Liu J, Zhou J, Wang S, Xiang H. Complete Genome Sequence of Haloarcula hispanica, a Model Haloarchaeon for Studying Genetics, Metabolism, and Virus-Host Interaction. I Bacteriol 2011; 193:6086-6087. PubMed doi:10.1128/|B.05953$\underline{11}$

3. Wang X, Greenfield P, Li D, Hendry P, Volk H, Sutherland TD. Complete Genome Sequence of a Nonculturable Methanococcus maripaludis Strain Extracted in a Metagenomic Survey of Petroleum Reservoir Fluids. I Bacteriol 2011; 193:5595. PubMed doi:10.1128/JB.05835-11

4. Park JY, Kim S, Kim SM, Cha SH, Lim SK, Kim J. Complete Genome Sequence of MultidrugResistant Acinetobacter baumannii Strain 1656-2, Which Forms Sturdy Biofilm. J Bacteriol 2011; 193:6393-6394. PubMed doi:10.1128/]B.0610911

5. Toh H, Sharma VK, Oshima K, Kondo S, Hattori M, Ward FB, Free A, Taylor TD. Complete Genome Sequences of Arcobacter butzleri ED-1 and Arcobacter sp. Strain L, Both Isolated from a Microbial Fuel Cell. J Bacteriol 2011; 193:64116412. PubMed doi:10.1128/JB.06084-11
6. Zhang A, Yang M, Hu P, Wu J, Chen B, Hua Y, Jun $\mathrm{Y}$, Chen $\mathrm{H}$, Xiao J, Jin M. Comparative Genomic Analysis of Streptococcus suis reveals significant genomic diversity among different serotypes. 2011; 12:523.

7. Stynen APR, Lage AP, Moore RJ, Rezende AM, de Resende VDS, Ruy PC, Daher N, Resende DM, de Almeida SS, Soares SC, et al. Complete Genome Sequence of Type Strain Campylobacter fetus subsp. venerealis NCTC 10354T. / Bacteriol 2011; 193:5871-5872. PubMed doi:10.1128//B.0585411

8. Kim HJ, Park JY, Han SH, Lee JH, Rong X, McSpadden Gardener BB, Park SK, Kim YC. Draft Genome Sequence of the Biocontrol Bacterium Chromobacterium sp. Strain C-61. I Bacteriol 2011; 193:6803-6804. PubMed doi:10.1128/JB.06191-11

9. Chen Y, Strain EA, Allard M, Brown EW. Genome Sequence of Cronobacter sakazakii E899, a Strain Associated with Human Illness. I Bacteriol 2011; 193:5861. PubMed doi:10.1128/JB.05913-11

10. Mancini S, Abicht HK, Karnachuk OV, Solioz M. Genome Sequence of Desulfovibrio sp. A2, a Highly Copper Resistant, Sulfate-Reducing Bacterium Isolated from Effluents of a Zinc Smelter at the Urals. J Bacteriol 2011; 193:67936794. PubMed doi:10.1128//B.06019-11

11. Koblížek $M$, Janouškovec J, Oborník $M$, Johnson JH, Ferriera S, Falkowski PG. Genome Sequence of the Marine Photoheterotrophic Bacterium 
Erythrobacter sp. Strain NAP1. J Bacteriol 2011;

193:5881-5882. PubMed doi:10.1128/JB.0584511

12. Yang $H$, Liao $Y$, Wang B, Lin $Y$, Pan L. Genome Sequence of Escherichia coli XH140A, Which Produces I-Threonine. J Bacteriol 2011; 193:6090-6091. PubMed doi:10.1128/JB.0601111

13. Yang $\mathrm{H}$, Liao $\mathrm{Y}$, Wang B, Lin $\mathrm{Y}$, Pan L. Draft Genome Sequence of Escherichia coli XH001, a Producer of I-Threonine in Industry. J Bacteriol 2011; 193:6406-6407. $\underline{\text { PubMed }}$ doi:10.1128/JB.06099-11

14. Jordan IK, Conley AB, Antonov IV, Arthur RA, Cook ED, Cooper GP, Jones BL, Knipe KM, Lee KJ, Liu X, et al. Genome Sequences for Five Strains of the Emerging Pathogen Haemophilus haemolyticus. J Bacteriol 2011; 193:5879-5880. PubMed doi:10.1128/JB.05863-11

15. Gupta HK, Singh A, Sharma R. Genome Sequence of Idiomarina sp. Strain A28L, Isolated from Pangong Lake, India. J Bacteriol 2011; 193:5875-5876. PubMed doi:10.1128/JB.05648$\underline{11}$

16. Liu L, Li Y, Zhang J, Zhou Z, Liu J, Li X, Zhou J, Du G, Wang L, Chen J. Complete Genome Sequence of the Industrial Strain Ketogulonicigenium vulgare WSH-001. J Bacteriol 2011; 193:6108-6109. PubMed doi:10.1128/JB.06007-11

17. Svenning MM, Hestnes AG, Wartiainen I, Stein LY, Klotz MG, Kalyuzhnaya MG, Spang A, Bringel F, Vuilleumier S, Lajus A, et al. Genome Sequence of the Arctic Methanotroph Methylobacter tundripaludum SV96. J Bacteriol 2011; 193:6418-6419. PubMed doi:10.1128//B.05380-11

18. Ishii S, Tago K, Nishizawa T, Oshima K, Hattori M, Senoo K. Complete Genome Sequence of the Denitrifying and N2O-Reducing Bacterium Pseudogulbenkiania sp. Strain NH8B. J Bacteriol 2011; 193:6395-6396. PubMed doi:10.1128/JB.06127-11

19. Tada T, Kitao T, Miyoshi-Akiyama T, Kirikae T. Genome Sequence of Multidrug-Resistant Pseudomonas aeruginosa NCGM1179. J Bacteriol 2011; 193:6397. PubMed doi:10.1128/JB.0612911

20. Park JY, Han SH, Lee JH, Han YS, Lee YS, Rong X, McSpadden Gardener BB, Park HS, Kim YC. Draft Genome Sequence of the Biocontrol Bacterium Pseudomonas putida B001, an Oligotrophic
Bacterium That Induces Systemic Resistance to Plant Diseases. J Bacteriol 2011; 193:6795-6796. PubMed doi:10.1128/JB.06217-11

21. Tang H, Yu H, Li Q, Wang X, Gai Z, Yin G, Su F, Tao F, Ma C, Xu P. Genome Sequence of Pseudomonas putida Strain B6-2, a Superdegrader of Polycyclic Aromatic Hydrocarbons and Dioxin-Like Compounds. J Bacteriol 2011; 193:6789-6790. PubMed doi:10.1128/JB.0620111

22. Chen M, Yan Y, Zhang W, Lu W, Wang J, Ping S, Lin M. Complete Genome Sequence of the Type Strain Pseudomonas stutzeri CGMCC 1.1803. J Bacteriol 2011; 193:6095. PubMed doi:10.1128/JB.06061-11

23. Li Z, Wu S, Bai X, Liu Y, Lu J, Liu Y, Xiao B, Lu X, Fan L. Genome Sequence of the Tobacco Bacterial Wilt Pathogen Ralstonia solanacearum. J Bacteriol 2011; 193:6088-6089. PubMed doi:10.1128/JB.06009-11

24. Gupta HK, Gupta RD, Singh A, Chauhan NS, Sharma R. Genome Sequence of Rheinheimera sp. Strain A13L, Isolated from Pangong Lake, India. J Bacteriol 2011; 193:5873-5874. PubMed doi:10.1128/JB.05636-11

25. Gai Z, Wang X, Tang H, Tai C, Tao F, Wu G, Xu $P$. Genome Sequence of Sphingobium yanoikuyae XLDN2-5, an Efficient Carbazole-Degrading Strain. / Bacteriol 2011; 193:6404-6405. PubMed doi:10.1128/JB.06050-11

26. Mancini S, Abicht HK, Karnachuk OV, Solioz M. Genome Sequence of Desulfovibrio sp. A2, a Highly Copper Resistant, Sulfate-Reducing Bacterium Isolated from Effluents of a Zinc Smelter at the Urals. J Bacteriol 2011; 193:67936794. PubMed doi:10.1128//B.06019-11

27. Su F, Xu K, Zhao B, Tai C, Tao F, Tang H, Xu P. Genome Sequence of the Thermophilic Strain Bacillus coagulans XZL4, an Efficient PentoseUtilizing Producer of Chemicals. J Bacteriol 2011; 193:6398-6399. PubMed doi:10.1128/JB.06157$\underline{11}$

28. Liu L, Li Y, Zhang J, Zou W, Zhou Z, Liu J, Li X, Wang L, Chen J. Complete Genome Sequence of the Industrial Strain Bacillus megaterium WSH002. J Bacteriol 2011; 193:6389-6390. PubMed doi:10.1128/JB.06066-11

29. Su F, Hua D, Zhang Z, Wang X, Tang H, Tao F, Tai C, Wu Q, Wu G, Xu P. Genome Sequence of Bacillus pumilus S-1, an Efficient IsoeugenolUtilizing Producer for Natural Vanillin. I Bacteriol 
2011; 193:6400-6401. PubMed

doi:10.1128/JB.06170-11

30. Abicht HK, Mancini S, Karnachuk OV, Solioz M. Genome Sequence of Desulfosporosinus sp. $\mathrm{O}^{\top}$, an Acidophilic Sulfate-Reducing Bacterium from Copper Mining Waste in Norilsk, Northern Siberia. J Bacteriol 2011; 193:6104-6105. PubMed doi:10.1128/JB.06018-11

31. Jung MJ, Roh SW, Kim MS, Whon TW, Bae JW. Genome Sequence of Lentibacillus jeotgali Grbi ${ }^{\top}$, Isolated from Traditional Korean Salt-Fermented Seafood. J Bacteriol 2011; 193:6414-6415. PubMed doi:10.1128/JB.06139-11

32. Nam SH, Kim A, Choi SH, Kang A, Kim DW, Kim RN, Kim DS, Park HS. Genome Sequence of Leuconostoc carnosum KCTC 3525. J Bacteriol 2011; 193:6100-6101. $\underline{\text { PubMed }}$ doi:10.1128/JB.06028-11

33. Buchrieser C, Rusniok C, Garrido P, Hain T, Scortti M, Lampidis R, Kärst U, Chakraborty T, Cossart P, Kreft J, et al. Complete Genome Sequence of the Animal Pathogen Listeria ivanovii, Which Provides Insights into Host Specificities and Evolution of the Genus Listeria. J Bacteriol 2011; 193:6787-6788. $\underline{\text { PubMed }}$ doi:10.1128/JB.06120-11

34. Beneduzi A, Campos S, Ambrosini A, de Souza R, Granada C, Costa P, Arruda L, Moreira F, Vargas LK, Weiss V, et al. Genome Sequence of the Diazotrophic Gram-Positive Rhizobacterium Paenibacillus riograndensis SBR5 ${ }^{\top}$. J Bacteriol 2011; 193:6391-6392. PubMed doi:10.1128/JB.06100-11

35. Yu B, Su F, Wang L, Xu K, Zhao B, Xu P. Draft Genome Sequence of Sporolactobacillus inulinus Strain CASD, an Efficient d-Lactic Acid-Producing Bacterium with High-Concentration Lactate Tolerance Capability. J Bacteriol 2011; 193:58645865. PubMed doi:10.1128/JB.05934-11

36. Shahinas D, Tamber GS, Arya G, Wong A, Lau R, Jamieson F, Ma JH, Alexander DC, Low DE, Pillai DR. Whole-Genome Sequence of Streptococcus pseudopneumoniae Isolate IS7493. I Bacteriol 2011; 193:6102-6103. PubMed doi:10.1128//B.06075-11

37. Geng J, Huang SC, Li S, Hu S, Chen YYM. Complete Genome Sequence of the Ureolytic Streptococcus salivarius Strain 57.I. / Bacteriol 2011; 193:5596-5597. PubMed doi:10.1128/JB.05670-11

38. Heng NCK, Haji-Ishak NS, Kalyan A, Wong AYC, Lovrić M, Bridson JM, Artamonova J, Stanton JAL,
Wescombe PA, Burton JP, et al. Genome Sequence of the Bacteriocin-Producing Oral Probiotic Streptococcus salivarius Strain M18. J Bacteriol 2011; 193:6402-6403. PubMed doi:10.1128/JB.06001-11

39. Zhang A, Yang M, Hu P, Wu J, Chen B, Hua Y, Jun Y, Chen H, Xiao J, Jin M: Comparative Genomic Analysis of Streptococcus suis reveals significant genomic diversity among different serotypes. 2011, 12:523.

40. Benomar N, Abriouel H, Lee H, Cho GS, Huch M, Pulido RP, Holzapfel WH, Gálvez A, Franz CMAP. Genome Sequence of Weissella thailandensis fsh4-2. J Bacteriol 2011; 193:5868. PubMed doi:10.1128/JB.05883-11

41. Guo Z, Chen P, Ren P, Kuang S, Zhou Z, Li Z, Liu M, Shi D, Xiao Y, Wang X, et al. Genome Sequence of Duck Pathogen Mycoplasma anatis Strain 1340. J Bacteriol 2011; 193:5883-5884. PubMed doi:10.1128/JB.05891-11

42. Chu $Y$, Gao $P$, Zhao $P$, He $Y$, Liao N, Jackman $S$, Zhao Y, Birol I, Duan X, Lu Z. Genome Sequence of Mycoplasma capricolum subsp.

capripneumoniae Strain M1601. J Bacteriol 2011; 193:6098-6099. PubMed doi:10.1128/JB.0598011

43. Calcutt MJ, Foecking MF. Genome Sequence of Mycoplasma putrefaciens Type Strain KS1. J

Bacteriol 2011; 193:6094. PubMed doi:10.1128/JB.06051-11

44. Cerdeira LT, Pinto AC, Schneider MPC, de Almeida SS, dos Santos AR, Barbosa EGV, Ali A, Barbosa MS, Carneiro AR, Ramos RTJ, et al. Whole-Genome Sequence of Corynebacterium pseudotuberculosis PAT10 Strain Isolated from Sheep in Patagonia, Argentina. / Bacteriol 2011; 193:6420-6421. PubMed doi:10.1128/JB.06044$\underline{11}$

45. Bottacini F, Dal Bello F, Turroni F, Milani C, Duranti S, Foroni E, Viappiani A, Strati F, Mora D, van Sinderen D, et al. Complete Genome Sequence of Bifidobacterium animalis subsp. lactis BLC1. J Bacteriol 2011; 193:6387-6388. PubMed doi:10.1128/JB.06079-11

46. Guinane CM, Barrett E, Fitzgerald GF, van Sinderen D, Ross RP, Stanton C. Genome Sequence of Bifidobacterium breve DPC 6330, a Strain Isolated from the Human Intestine. J Bacteriol 2011; 193:6799-6800. PubMed doi:10.1128/JB.06196-11

47. Park SK, Roh SW, Whon TW, Bae JW. Genome Sequence of Brachybacterium squillarum M-6-3 ${ }^{\top}$, 
Isolated from Salt-Fermented Seafood. / Bacteriol

2011; 193:6416-6417. PubMed

doi:10.1128/JB.06183-11

48. Hayano-Kanashiro C, López-Arredondo DL, CruzMorales P, Alcaraz LD, Olmedo G, BaronaGómez F, Herrera-Estrella L. First Draft Genome Sequence of a Strain from the Genus Citricoccus. J Bacteriol 2011; 193:6092-6093. PubMed doi:10.1128/JB.06045-11

49. Lv Y, Wu Z, Han S, Lin Y, Zheng S. Genome Sequence of Corynebacterium glutamicum S9114, a Strain for Industrial Production of Glutamate. J

Bacteriol 2011; 193:6096-6097. PubMed doi:10.1128/|B.06074-11

50. Kim J, Roh SW, Bae JW. Draft Genome Sequence of Dietzia alimentaria 72T, Belonging to the Family Dietziaceae, Isolated from a Traditional Korean Food. J Bacteriol 2011; 193:6791. PubMed doi:10.1128/JB.06229-11

51. González-Pérez M, Murcia MI, Landsman D, Jordan IK, Mariño-Ramírez L. Genome Sequence of the Mycobacterium colombiense Type Strain, CECT 3035. J Bacteriol 2011; 193:5866-5867. PubMed doi:10.1128/JB.05928-11

52. Miyoshi-Akiyama T, Matsumura K, Kobayashi N, Maeda S, Kirikae T. Genome Sequence of Clinical Isolate Mycobacterium tuberculosis NCGM2209.

J Bacteriol 2011; 193:6792. PubMed doi:10.1128/JB.06233-11

53. Tao F, Zhao P, Li Q, Su F, Yu B, Ma C, Tang H, Tai C, Wu G, Xu P. Genome Sequence of Rhodococcus erythropolis XP, a Biodesulfurizing Bacterium with Industrial Potential. J Bacteriol 2011; 193:6422-6423. PubMed

54. Xiao J, Luo Y, Xu J. Genome Sequence of Serinicoccus profundi, a Novel Actinomycete Isolated from Deep-Sea Sediment. / Bacteriol 2011 ; 193:6413. PubMed doi:10.1128/JB.0611911

55. Zhong $Y$, Chang X, Cao XJ, Zhang Y, Zheng $H$, Zhu Y, Cai C, Cui Z, Zhang Y, Li YY, et al. Comparative proteogenomic analysis of the Leptospira interrogans virulence-attenuated strain IPAV against the pathogenic strain 56601. Cell Res 2011; 21:1210-1229. PubMed doi:10.1038/cr.2011.46

56. Kim MS, Whon TW, Roh SW, Shin NR, Bae JW. Draft Genome Sequence of Bacteroides faecis MAJ27 $7^{\top}$, a Strain Isolated from Human Feces. J Bacteriol 2011; 193:6801-6802. PubMed doi:10.1128//B.06210-11
57. Lanzarotti E, Pellizza L, Bercovich A, Foti M, Coria SH, Vazquez SC, Ruberto L, Hernández EA, Dias RL, Mac Cormack WP, et al. Draft Genome Sequence of Bizionia argentinensis, Isolated from Antarctic Surface Water. J Bacteriol 2011; 193:6797-6798. PubMed doi:10.1128/JB.0624511

58. Touchon M, Barbier P, Bernardet JF, Loux V, Vacherie B, Barbe V, Rocha EPC, Duchaud E. Complete Genome Sequence of the Fish Pathogen Flavobacterium branchiophilum. Appl Environ Microbiol 2011; 77:7656-7662. PubMed doi:10.1128/AEM.05625-11

59. Oh C, Kwon YK, Heo SJ, De Zoysa M, Affan A, Lee $\mathrm{Y}$, Lee J, Choi YU, Park HS, Jung KH, et al. Complete Genome Sequence of Strain S85, a Novel Member of the Family Flavobacteriaceae. J Bacteriol 2011; 193:6107. PubMed doi:10.1128/JB.05993-11

60. Swithers KS, DiPippo JL, Bruce DC, Detter C, Tapia R, Han S, Saunders E, Goodwin LA, Han J, Woyke T, et al. Genome Sequence of Thermotoga sp. Strain RQ2, a Hyperthermophilic Bacterium Isolated from a Geothermally Heated Region of the Seafloor near Ribeira Quente, the Azores. J Bacteriol 2011; 193:5869-5870. PubMed doi:10.1128/JB.05923-11

61. Futagami T, Mori K, Yamashita A, Wada S, Kajiwara Y, Takashita H, Omori T, Takegawa K, Tashiro K, Kuhara S, et al. Genome Sequence of the White Koji Mold Aspergillus kawachii IFO 4308, Used for Brewing the Japanese Distilled Spirit Shochu. Eukaryot Cell 2011; 10:1586-1587. PubMed doi:10.1128/EC.05224-11

62. Varshney RK, Chen W, Li Y, Bharti AK, Saxena RK, Schlueter JA, Donoghue MTA, Azam S, Fan G, Whaley AM, et al.: Draft genome sequence of pigeonpea (Cajanus cajan), an orphan legume crop of resource-poor farmers. Nat Biotech 2011, advance online publication.

63. Yip CCY, Lau SKP, Woo PCY, Chan KH, Yuen KY. Complete Genome Sequence of a Coxsackievirus A22 Strain in Hong Kong Reveals a Natural Intratypic Recombination Event. / Virol 2011; 85:12098-12099. $\underline{\text { PubMed }}$ doi:10.1128/JVI.05944-11

64. Petrovski S, Tillett D, Seviour RJ. Genome sequence and characterization of the related Gordonia phages GTE5 and GRU1 and their use as potential biocontrol agents. Appl Environ Microbiol 2011; 77:1. PubMed 
65. Kim EB, Fang $X$, Fushan AA, Huang Z, Lobanov AV, Han L, Marino SM, Sun X, Turanov AA, Yang $P$, et al. Genome sequencing reveals insights into physiology and longevity of the naked mole rat. Nature $2011 ;$ 479:223-227. PubMed doi:10.1038/nature10533

66. Dehghan S, Seto J, Hudson NR, Robinson CM, Jones MS, Dyer DW, Chodosh J, Seto D. Complete Genome Sequence of Human Adenovirus Prototype 17. J Virol 2011; 85:1154011541. PubMed doi:10.1128//VI.06051-11
67. Yan G, Zhang G, Fang X, Zhang Y, Li C, Ling F, Cooper DN, Li Q, Li Y, van Gool AJ, et al. Genome sequencing and comparison of two nonhuman primate animal models, the cynomolgus and Chinese rhesus macaques. Nat Biotechnol 2011; 29:1019-1023. PubMed doi:10.1038/nbt.1992

68. Chen J, Wang C, Shi H, Qiu HJ, Liu S, Shi D, Zhang X, Feng L. Complete Genome Sequence of a Chinese Virulent Porcine Epidemic Diarrhea Virus Strain. J Virol 2011; 85:11538-11539. PubMed doi:10.1128//VI.06024-11 